ORIGINAL ARTICLE

\title{
An audit of splenectomies in a teaching hospital in North India. Are postsplenectomy guidelines being complied with?
}

\author{
M Deodhar, N Kakkar
}

J Clin Pathol 2004;57:407-410. doi: 10.1136/jcp.2003.013896

See end of article for authors' affiliations Correspondence to: Dr N Kakkar, Department of Pathology, Christian Medical College and Hospital, Ludhiana-141 008, Punjab, India; n_kakkar@satyam.net.in

Accepted for publication 28 November 2003

\begin{abstract}
Aims: Patients with an absent or dysfunctional spleen are at risk of infection by encapsulated and other bacteria. Overwhelming postsplenectomy infection (OPSI) causes most concern because it can result in significant mortality. A retrospective review of splenectomised patients in a tertiary care setting over an eight year period was carried out to determine whether current postsplenectomy guidelines were being followed.

Methods: The cases were identified from the medical records and pathology files and data such as the reason for splenectomy, the preventive measures taken regarding vaccination, and antibiotic prophylaxis, together with their documentation in the discharge notes were assessed.

Results: Fiffy six patients were studied. Trauma, both blunt and penetrating, was the most common reason for splenectomy. Thirty six patients received pneumococcal vaccination, with 20 patients having no mention of vaccination in their case notes. The discharge notes of 50 patients mentioned their splenectomised status; however, documentation of vaccination details in the discharge summary was poor, with only three patients having the relevant information recorded. Documentation of the need for future vaccination and precautions required in the asplenic condition was also lacking. Nine patients had postsplenectomy complications, although there were no cases of OPSI.

Conclusions: Adherence to standard guidelines for the management of splenectomised patients was unsatisfactory. There is a need for an improvement of the vaccination rate and careful documentation of this important health risk in the discharge summaries. Maintenance of a splenectomy registry could aid in optimising the management of these patients.
\end{abstract}

$\mathrm{T}$ he role of the spleen in the body's immune response to a variety of infections and the serious consequences of its removal have been increasingly recognised over the past $40-50$ years. ${ }^{1}$ The asplenic condition results in an increased risk of life threatening sepsis as a result of encapsulated and Gram negative organisms, and carries considerable mortality. The overall incidence of septicaemia is low, but death rates from overwhelming post splenectomy infection (OPSI) have been reported to be 600 times greater than that for the general population, with an ominous estimated lifetime risk for OPSI of approximately $5 \%{ }^{2}$ With increasing awareness of the risk associated with the asplenic state, splenic salvage surgery is now gaining popularity. ${ }^{3}$ However, there are many clinical indications that warrant a total splenectomy, such as extensive splenic trauma or haematological disorders, resulting in a large population of asplenic patients.

"Patient education and counselling at the time of splenectomy is just as important as appropriate vaccination and antibiotic prophylactic measures"

Vaccination strategies, which started with the use of the pneumococcal vaccine, have been modified since their inception in the 1970 s to circumvent the emergence of antibiotic resistant strains and the recognition of other pathogens that cause infections in splenectomised patients. ${ }^{5}$ Thus, patient education and counselling at the time of splenectomy is just as important as appropriate vaccination and antibiotic prophylactic measures. ${ }^{6}$ The past decade has seen increasing interest among clinicians regarding the appropriate management for the prevention and treatment of infections in both asplenic and hyposplenic individuals.
We carried out our study to assess the enforcement of postsplenectomy guidelines in our hospital.

\section{MATERIAL AND METHODS}

Our study was carried out in the departments of surgery and pathology, Christian Medical College and Hospital, Ludhiana, India, which is a 800 bed teaching institution.

A retrospective audit of all patients who underwent splenectomy for any indication, emergency or elective, over an eight year period from 1996 to 2003 was performed. The patients were identified by the International Classification of Diseases coding in the medical records section and the biopsy index in the pathology section. Their case notes were reviewed for the reason for splenectomy, associated injuries (in case of a traumatic aetiology), any postsplenectomy complications, and the follow up status. The preoperative and postoperative vaccination details and the documentation of splenectomy as a health risk in the discharge summaries were also recorded. Patients undergoing spleen conserving surgery, in addition to those who died during hospitalisation after the splenectomy, were excluded from our study.

\section{RESULTS AND ANALYSIS}

In the eight year study period, 56 patients underwent splenectomy in our hospital. These included 49 males and seven females (male to female ratio, $7: 1$ ), with an age range of 3-82 years (mean age, 33.5). Fifty one splenectomies were performed as emergency procedures for varying grades of splenic trauma, whereas elective surgery was performed in

Abbreviation: OPSI, overwhelming postsplenectomy infection 
Table 1 Reasons for splenectomy among the 56 patients in our study group

\begin{tabular}{ll}
\hline Indication for splenectomy & Number of patients \\
\hline Traumatic & \\
Blunt trauma & 47 \\
Penetrating trauma & 3 \\
latrogenic & 1 \\
Non-haematological & 1 \\
Portal hypertension & 1 \\
Carcinoma, stomach & 1 \\
Carcinoma, pancreas & 1 \\
Carcinoma, kidney & 1 \\
Haematological & 1 \\
B cell non-Hodgkin lymphoma & \\
\hline
\end{tabular}

five patients who had non-traumatic indications-both haematological and non-haematological. Table 1 lists the details.

Along with the splenic injury, 23 patients also suffered from skeletal injuries, including fracture of the pelvis, ribs, and long bones, whereas 11 patients had associated soft tissue and visceral trauma.

Thirty six of the 56 patients included in our study received prophylactic vaccination. Only pneumococcal (PNEUMOVAC) vaccination was used for prophylaxis. None of the patients received vaccination for Neisseria meningitides or Haemophilus influenzae. Thirty four patients received the vaccine postoperatively and two were vaccinated in the preoperative period. Both the patients who received preoperative vaccine prophylaxis underwent elective splenectomies for non-traumatic indications. Postsplenectomy vaccination was given within a time frame of $1-26$ days, with 17 of the patients receiving the vaccine within 48 hours of surgery and three patients being vaccinated more than two weeks after splenectomy. The case records of 20 patients did not reveal documentation of vaccination.

Fifty three of the 56 patients received a discharge summary; 50 of these patients had their splenectomised status mentioned in the discharge notes. The vaccination status was documented in only three patients. The discharge summary in only two patients mentioned the need for future vaccination, and two patients were advised regarding the necessity of lifelong antibiotic prophylaxis.

None of the patients was given a card highlighting the precautions to be taken after splenectomy, as is recommended.

The follow up period ranged from seven to 790 days (mean, 116.1). Four patients were lost to follow up. Nine patients had postoperative complications, which were related to the surgical procedure itself in five. However, none of the complications qualified as overwhelming post splenectomy infection (OPSI). Table 2 lists the complications encountered.

Table 2 The complications encountered in nine patients after splenectomy

\begin{tabular}{ll}
\hline Postsplenectomy complication & Number of patients \\
\hline Acute renal failure & 2 \\
Wound infection* & 2 \\
Subacute intestinal obstruction* & 1 \\
Incisional hernia* & 1 \\
Hepatic encephalopathy & 1 \\
Subphrenic abscess* & 1 \\
Malaria (Plasmodium falciparum) & 1 \\
\hline
\end{tabular}

*Complications related to the surgical procedure itself.

\section{DISCUSSION}

The asplenic state is a health risk increasingly being recognised by health professionals all over the world. Infection in patients with an absent or dysfunctional spleen remains largely preventable. Many measures have been proposed to reduce the risk of sepsis after splenectomy, which include chemoprophylaxis, immunoprophylaxis, and patient/clinician education. ${ }^{6}$

Other than the known susceptibility of splenectomised individuals to encapsulated bacteria (Streptococcus pneumoniae, $N$ meningitides, and $H$ influenziae $),{ }^{7}$ there is evidence to suggest that other organisms, such as Gram negative bacteria and Capnocytophaga canimorsus, ${ }^{2}$ in addition to intraerythrocytic parasites, such as babesia and plasmodium, can cause infections and carry considerable morbidity. ${ }^{8}$

The pneumococcal vaccine, first introduced in the 1970s, presently includes 23 serotypes responsible for approximately $88 \%$ of pneumococcal infections. However, the most virulent pneumococcal serotypes tend to be the least immunogenic, and there is clear evidence that vaccine efficacy is poorest in younger patients who, unfortunately, also comprise the highest risk group..$^{10}$

Pneumococcal vaccine protection rates are $70 \%$ in a healthy immunocompetent host because approximately $10 \%$ of possible antibody responses to individual antigens do not occur. ${ }^{9}$ The British Committee for Standards in Haematology first published guidelines for the prevention and treatment of infection in asplenic and hyposplenic patients in $1996 .{ }^{11}$ The salient aspects of these guidelines are related to anti-infective prophylaxis, immunisation schedules, and treatment of confirmed or suspected infections. These guidelines have recently been updated with minor amendments. A newer recommendation has been to include the use of the meningococcal vaccine in previously non-immunised patients and a need to consider the use of the seven valent pneumococcal vaccine. ${ }^{12}$

However, vaccination should not result in a false sense of security because vaccine failures are frequent. There are relatively fewer studies previously carried out to determine the vaccination status and use of antibiotic prophylaxis in splenectomised subjects.

Brigden et al performed an audit on 164 hospitalised patients who underwent splenectomies in British Columbia, Canada. ${ }^{13}$ Sixty eight percent of patients had received pneumococcal vaccination. Of the 55 patients who underwent elective surgery, only 11 received preoperative vaccination. Most (95\%) of the patients had documentation of their splenectomised status in their discharge summaries. However, documentation of the vaccination status, the need for future vaccination, and information on future infection risks was inadequate, with only $21 \%, 6 \%$, and $5 \%$ of the discharge notes giving this information, respectively.

Ramachandra et al studied postsplenectomy prophylaxis status in 76 patients from a general hospital. ${ }^{14}$ Fifty five of the patients had received vaccination, whereas 48 were discharged on prophylactic antibiotics. Eighty one percent of the surviving patients in their study had adequate communication with the general practitioner regarding their splenectomised status. In their study, patients undergoing non-elective splenectomy were less likely to be vaccinated or to receive prophylactic antibiotics.

In another study, among 28 patients who underwent splenectomy in a district hospital, trauma was the indication in only four patients. The remaining 24 patients either had haematological indications or had their spleen removed as part of other abdominal surgery. Most patients were prescribed pneumococcal vaccine (24) or prophylactic antibiotics $(26) .{ }^{15}$ 


\section{Take home messages}

- Similar to other reports, we found that adherence to standard guidelines for the management of splenectomised patients was unsatisfactory

- The vaccination rate in these patients needs to be improved

- The discharge summaries of these patients should contain careful documentation of this important health risk

- Maintenance of a splenectomy registry could aid in optimising the management of these patients

A study to evaluate postsplenectomy vaccine prophylaxis in Lothian, UK, revealed that $80.6 \%$ of the patients were vaccinated against $S$ pneumoniae. The vaccination rates for $H$ influenzae and $N$ meningitidis were $65.9 \%$ and $48.2 \%$, respectively. Three quarters of the splenectomised patients were prescribed longterm antibiotic prophylaxis. ${ }^{16}$

Ejstrud et al evaluated pneumococcal vaccine prophylaxis in 555 patients who underwent splenectomy over a 10 year period. ${ }^{17}$ Vaccination rates were $62 \%$, with patients undergoing splenectomy during cancer surgery or after inadvertent intraoperative trauma particularly at risk of not receiving vaccination. Only $23 \%$ of the patients were vaccinated at the appropriate time, with the splenectomy status unrecorded in $10 \%$ of cases. Vaccination status was mentioned in $35 \%$ of the discharge notes, whereas only $2 \%$ mentioned the precautions to be taken for asplenic patients.

"Although 50 of the 53 discharge summaries issued mentioned the splenectomised status of patients, only three mentioned the vaccination status"

Waghorn $^{18}$ studied data on 77 asplenic patients who developed OPSI by means of a questionnaire survey among microbiologists across the UK. Overall mortality with OPSI was $50 \%$. Only 24 patients had received prophylactic pneumococcal vaccination before OPSI. There were seven cases of pneumococcal vaccine failure. Accepted guidelines were not being followed, with only a few patients being adequately advised on antibiotic prophylaxis or other measures. Most similar published studies in patients who have undergone splenectomy have shown that standard guidelines regarding vaccination and antibiotic prophylaxis are not followed strictly. Our study has also shown inadequacies in the adherence to currently accepted postsplenectomy management strategies. The overall vaccination rate in our study was $64.3 \%$, which is comparable to most other reports. However, alarmingly, 20 of the 56 patients in our study did not receive vaccination. Most of the patients received the vaccination postoperatively, which is understandable, because most splenectomies were carried out as emergency procedures for splenic trauma. Although 50 of the 53 discharge summaries issued mentioned the splenectomised status of patients, only three mentioned the vaccination status. The need for future vaccination and antibiotic prophylaxis was also poorly documented. Documentation of extra caution to be taken by patients in the wake of any infections was poorly documented. Similarly, there was no record in the case notes to suggest that the patients were given a card and/or bracelet specifying their asplenic condition, information that could be vital for their general practitioner. Medical personnel dealing with asplenic patients need to be more vigilant in ensuring that prophylactic vaccination and antibiotic schedules are followed and in educating patients about their asplenic state. Maintenance of a hospital splenectomy registry can aid in keeping track of the health status of this potential risk group. A protocol sheet could be included in the case notes of the patient and the subsequent follow up should be documented on it. This should help to ensure compliance with regard to postsplenectomy guidelines (appendix 1).

\section{ACKNOWLEDGEMENTS}

The authors wish to thank Mr R Singh and his staff for their assistance in the retrieval of the case notes for this study.

\section{Authors' affiliations}

M Deodhar, Department of Surgery, Christian Medical College and Hospital, Ludhiana-141 008, Punjab, India

N Kakkar, Department of Pathology, Christian Medical College and Hospital

\section{APPENDIX 1 \\ SUGGESTED PROTOCOL FOR AFTERCARE OF THE SPLENECTOMISED PATIENT}

Table 3 Protocol sheet to be included in the patient's case notes

Name

Age

Sex

Hospital identification number

Splenectomy performed on (date)

Indication

Pneumococcal vaccination given (yes/no)

If vaccinated, vaccination given on (date)

Type of vaccine

Next booster due on (date)

Any other vaccination (meningococcal/influenza)

Date

Antibiotic prophylaxis (received/not received)

\section{Information to patients (check list)}

- Patient informed about risk of infections.

- Information on booster dose given.

- Educational booklet/leaflet given.

- Splenectomy card/bracelet.

- Education about risks of overseas travel.

- Importance of lifelong antibiotic prophylaxis.

\section{REFERENCES}

1 Shaw JHF, Print CG. Postsplenectomy sepsis. Br J Surg 1989:76:1074-81.

2 Lynch AM, Kapila R. Overwhelming postsplenectomy infection. Infect Dis Clin North Am 1996;4:693-707

3 Falk GL, Cregan PC, Kennedy CW. Splenic injury. An assessment of splenic conservation. Aust N Z J Surg 1991;61:354-7.

4 Dupuy DE, Raptopoulos V, Fink MP. Current concepts in splenic trauma. J Intensive Care Med 1995; 10:76-90.

5 Machesky K, Cushing R. Overwhelming post-splenectomy infection in a patient with penicillin resistant Streptococcus pneumoniae. Arch Fam Med 1998; 7:178-80.

6 White KS, Covington D, Churchill P, et al. Patient awareness of health precautions after splenectomy. Am J Infect Control 1991;19:36-41.

7 Brigden M, Pattullo A. Prevention and management of overwhelming postsplenectomy infection - an update. Crit Care Med 1999;27:836-42.

8 Styrt B. Infection associated with asplenia: risks, mechanisms and prevention. Am J Med 1990;88:33-42.

9 Butler J, Breiman R, Campbell J, et al. Pneumococcal polysaccharide vaccine efficacy: an evaluation of current recommendations. J Am Med Assoc 1993;270:1826-31. 
10 Shetty N, Aurora P, Ridgway G. Failure of anti-pneumococcal vaccine and prophylactic penicillin in a splenectomised patient. J Infect 1998;37:87-92.

11 Working Party of the British Committee for Standards in Hematology. Clinical haematology task force. Guidelines for the prevention and treatment of infection in patients with an absent or dysfunctional spleen. BMJ 1996;312:430-4

12 Working Party of the British Committee for Standards in Hematology Haemato-oncology task force. Update of guidelines for the prevention and treatment of infection in patients with an absent or dysfunctional spleen. Clin Med 2002;2:440-3.

13 Brigden M, Pattullo A, Brown G. Pneumococcal vaccine associated with splenectomy: the need for improved education, documentation, and the use of a practical checklist. Am J Hematol 2000;65:25-9.
14 Ramachandra J, Bond A, Ranaboldo C, et al. An audit of post-splenectomy prophylaxis - are we following the guidelines? Ann R Coll Surg Engl 2002;85:252-5.

15 Glass JM, Gilbert JM. Splenectomy in a general hospital. J R Soc Med 1996:89:199-201.

16 Pickering J, Campbell H. An audit of the vaccination and antibiotic prophylaxis practices amongst patients splenectomised in Lothian. Health Bull (Edinb) 2000;58:390-5.

17 Ejstrud P, Hansen JB, Andreasen DA. Prophylaxis against pneumococcal infection after splenectomy: a challenge for hospitals and primary care. Eur J Surg 1997;163:733-8.

18 Waghorn DJ. Overwhelming infection in asplenic patients: current best practice preventive measures are not being followed. J Clin Pathol 2001:54:214-18.

\section{Clinical Evidence-Call for contributors}

Clinical Evidence is a regularly updated evidence based journal available worldwide both as a paper version and on the internet. Clinical Evidence needs to recruit a number of new contributors. Contributors are health care professionals or epidemiologists with experience in evidence based medicine and the ability to write in a concise and structured way.

\section{Currently, we are interested in finding contributors with an interest in} the following clinical areas:

Altitude sickness; Autism; Basal cell carcinoma; Breast feeding; Carbon monoxide poisoning; Cervical cancer; Cystic fibrosis; Ectopic pregnancy; Grief/bereavement; Halitosis; Hodgkins disease; Infectious mononucleosis (glandular fever); Kidney stones; Malignant melanoma (metastatic); Mesothelioma; Myeloma; Ovarian cyst; Pancreatitis (acute); Pancreatitis (chronic); Polymyalgia rheumatica; Post-partum haemorrhage; Pulmonary embolism; Recurrent miscarriage; Repetitive strain injury; Scoliosis; Seasonal affective disorder; Squint; Systemic lupus erythematosus; Testicular cancer; Varicocele; Viral meningitis; Vitiligo However, we are always looking for others, so do not let this list discourage you.

\section{Being a contributor involves:}

- Appraising the results of literature searches (performed by our Information Specialists) to identify high quality evidence for inclusion in the journal.

- Writing to a highly structured template (about 2000-3000 words), using evidence from selected studies, within 6-8 weeks of receiving the literature search results.

- Working with Clinical Evidence Editors to ensure that the text meets rigorous epidemiological and style standards.

- Updating the text every eight months to incorporate new evidence.

- Expanding the topic to include new questions once every 12-18 months.

If you would like to become a contributor for Clinical Evidence or require more information about what this involves please send your contact details and a copy of your CV, clearly stating the clinical area you are interested in, to Claire Folkes (cfolkes@bmigroup.com).

\section{Call for peer reviewers}

Clinical Evidence also needs to recruit a number of new peer reviewers specifically with an interest in the clinical areas stated above, and also others related to general practice. Peer reviewers are health care professionals or epidemiologists with experience in evidence based medicine. As a peer reviewer you would be asked for your views on the clinical relevance, validity, and accessibility of specific topics within the journal, and their usefulness to the intended audience (international generalists and health care professionals, possibly with limited statistical knowledge). Topics are usually 2000-3000 words in length and we would ask you to review between 2-5 topics per year. The peer review process takes place throughout the year, and our turnaround time for each review is ideally 10-14 days.

If you are interested in becoming a peer reviewer for Clinical Evidence, please complete the peer review questionnaire at www.clinicalevidence.com or contact Claire Folkes(cfolkes@bmigroup.com). 